\title{
Hypoglycemic effect of sea buckthorn leaves supplementation in streptozotocin-induced diabetic rats
}

\author{
M. W. Kim, H. K. Han and S. S. Choi \\ Department of Food and Nutrition, Duksung Women's University, Seoul, Republic of Korea
}

People concern for preventing the chronic disease is more focused on nutraceutical or natural biocompound sources rather than medicinal therapeutic agents. Sea buckthorn has been reported to possess hypoglycemic property in normal mice ${ }^{(1)}$. In this study, we examined the effects of Hippophae rhamnoides L. (sea buckthorn: SBT) on the plasma blood glucose and cholesterol level in diabetic rats. Diabetes mellitus was induced in male Sprague-Dawley rats weighing 200-220 g by an injection of streptozotocin (STZ) dissolved in a citrate buffer into the tail vein at a dose of $45 \mathrm{mg} / \mathrm{kg}$ of body weight. Spargue-Dawley rats were fed an AIN-93 recommended diet and experimental groups were fed a modified diet containing $10 \%$ and $20 \%$ of SBT powder for 4 weeks. The experimental groups were divided into six groups, which consisted of normal (N)-control, N-SBT 10\%, N-SBT $20 \%$ treated groups, STZ-control, STZ-SBT 10\% and STZ-SBT $20 \%$ treated group. The rat body weight, aspartate aminotransferase activity and hematocrit values were measured. The concentrations of plasma blood glucose and cholesterol were analysed. Body weight losses were observed by diabetic groups, while the no diabetic rats gained weight. There were significant differences between the control group and the diabetic groups in the weight of kidney $(P<0.05)$. Aspartate aminotransferase activity was lower in the non-diabetic group compared to diabetic experimental groups. The blood glucose levels were significantly decreased in the STZ-SBT $10 \%$ group $(P<0.05)$. The cholesterol level of STZ-SBT $10 \%$ and STZ-SBT $20 \%$ were significantly lower than for the STZ-control group $(P<0.05)$. These results show that the supplementation of sea buckthorn may have favourable influence on reducing the blood glucose and cholesterol level in STZ-induced diabetic rats.

1. Zhang W, Zhao J, Wang J et al. (2010) Hypoglycemic effect of aqueous extract of seabuckthron (Hippophae rhamnoides L.) seed residues in streptozotocin-induced diabetic rats. Phytother Res 24, 228-232. 